\title{
Infections due to Carbapenem Resistant Enterobacteriaceae among Saudi Arabian Hospitalized Patients: A Matched Case-Control Study
}

\author{
M. A. Garbati, ${ }^{1}$ H. Sakkijha, ${ }^{2}$ and A. Abushaheen ${ }^{3}$ \\ ${ }^{1}$ Section of Infectious Diseases, Medical Specialties Department, King Fahad Medical City, P.O. Box 59046, Riyadh 11525, Saudi Arabia \\ ${ }^{2}$ Pulmonary and Critical Care Medicine Department, King Fahad Medical City, P.O. Box 59046, Riyadh 11525, Saudi Arabia \\ ${ }^{3}$ Scientific Research and Publication Center, King Fahad Medical City, P.O. Box 59046, Riyadh 11525, Saudi Arabia \\ Correspondence should be addressed to M. A. Garbati; musagarbati@yahoo.com
}

Received 15 December 2015; Accepted 13 March 2016

Academic Editor: Paola Di Carlo

Copyright (C) 2016 M. A. Garbati et al. This is an open access article distributed under the Creative Commons Attribution License, which permits unrestricted use, distribution, and reproduction in any medium, provided the original work is properly cited.

\begin{abstract}
Background. We conducted this case-control study to determine the risk factors and treatment outcome of infections due to carbapenem resistant Enterobacteriaceae in our institution. Methods. This is a matched case-control study of patients with infection due to carbapenem resistant Enterobacteriaceae (CRE) and carbapenem susceptible Enterobacteriaceae (CSE), from Riyadh, Saudi Arabia, between March 2012 and December 2013. Results. During this period, 29 cases and 58 controls were studied. The mean ages of the cases (55.4 years) and controls (54.7 years) were similar $(p=0.065)$. Cases had higher mean Charlson comorbidity index (CCI) (3.1) than controls (1.9), $p=0.026$. Several factors contributed to infection among the studied population. Prior uses of piperacillin-tazobactam, a carbapenem, a quinolone, and metronidazole were significantly associated with CRE infections. Nine of the cases died compared with 7 of the controls, $p=0.031$. Mortality was associated with advanced age, the presence of comorbidities, ICU stay, and receipt of invasive procedures. Conclusions. Infections due to CRE resulted in a significantly increased mortality. Combination antibiotic therapy was associated with reduced mortality. Properly designed randomized controlled studies are required to better characterize these findings.
\end{abstract}

\section{Introduction}

Carbapenems are antibiotics of last resort used to treat severe infections due to Gram-negative rods (GNR), such as those caused by extended spectrum $\beta$-lactamase- (ESBL-) producing organisms [1]. Unfortunately, resistance to these life-saving drugs has increasingly been reported among the most clinically relevant bacteria $[2,3]$, selective pressure being the major determinant [4]. Resistance to carbapenems is predominantly conferred by carbapenemases, such as oxacillinase- (OXA-) type enzymes and metallo$\beta$-lactamases (MBLs) of Imipenemase (IMP), New Delhi metallo- $\beta$-lactamases (NDM) and Verona, Italy, metallo$\beta$-lactamases (VIM) types, and serine carbapenemases of Klebsiella pneumoniae (KPC) type [3].

During the last decade increased prevalence of infections due to carbapenem resistant Enterobacteriaceae (CRE) has been reported [5]. Significant morbidity and mortality have been attributed to infections due to these isolates, especially bacteremia with attributable mortality rates of up to $50 \%$ compared with carbapenem susceptible Enterobacteriaceae (CSE) [6-9]. This is mainly related to delays in providing effective therapy. Previous case-control studies have identified various risk factors associated with carbapenem resistant Klebsiella pneumoniae (CRKP) infections [10, 11] that included antibiotic exposure, intensive care unit (ICU) stay, and poor functional status. Recently, Hussein et al. [12] reported that prior exposure to any antibiotic increases the chances of infection with CRE (OR 3.3 (95\% CI 1.3-8.5), $p=$ 0.012 ).

The optimal treatment of infections due to CRE is yet unknown. With few new antimicrobials under development, clinicians have resorted to using older, previously discarded antimicrobials, such as colistin and tigecycline, alone or in 
combination with carbapenems, aminoglycosides, and fosfomycin, to treat these infections $[13,14]$. This approach has recently led to the emergence of organisms with resistance to antibiotics from all known classes, including the polymyxins [15]. Current clinical evidence for treatment guidance is limited and based on retrospective observational studies and case reports $[15,16]$. A recent ICU outbreak of Klebsiella oxytoca infection revealed that monotherapy tended to be associated with higher mortality compared with combination therapy (60\% versus $16.6 \%, p=0.07)$ [17].

As observed, the threat of infections due to multidrug resistant Gram-negative bacteria (MDR GNB) is a growing concern across the Middle East but the risk factors for acquisition, as well as treatment outcomes, have not been previously characterized. Thus, the aim of this study was to identify the prevalence of infections due to CRE, in relation to the risk factors and outcome of treatment.

\section{Methods}

2.1. Study Site and Population. A matched case-control study was conducted on adult patients attending King Fahad Medical City (KFMC), Riyadh, between March 2012 and December 2013.

\subsection{Subject Definitions and Study Protocol}

2.2.1. Cases. Patients were enrolled as cases if they had a new diagnosis of hospital-acquired infection with CRE.

2.2.2. Controls. Patients were enrolled as controls if they had a new diagnosis of hospital-acquired infection with at least one culture positive for CSE. For each patient with CRE infection, we selected two controls matched for anatomic site of infection and the causative organism.

The study focused on the first episode of hospitalacquired infection even if recurrent infections occurred. All data were captured prospectively, and no additional tests were performed for the purpose of this study. Identification of cases for inclusion in the study was done consecutively and triggered from the microbiology laboratory where any isolate of CRE (mainly Klebsiella pneumoniae, Escherichia coli, Enterobacter species, and Citrobacter species) was flagged by the personnel who alerted the investigators. Controls were selected consecutively from a list of all CSE isolated in the microbiology laboratory of our center during the study period and their data were extracted prospectively. Due to the rarity of infection with some of these organisms, controls were selected without randomization. All identified eligible controls were screened for inclusion.

\subsection{Microbiological Testing. Identification of infecting organ-} isms (CRE or CSE) was performed using routine microbiological methods. Susceptibility testing for meropenem and imipenem was performed by both the disc diffusion method and an automated broth microdilution method (bioMerieux, Vitek II, Hazelwood, MO, USA), according to manufacturer's instructions. Isolates from clinical specimens during the study period were identified as carbapenem resistant when imipenem and/or meropenem resistance was documented. Antibiotic susceptibility was interpreted as per criteria published by The Clinical and Laboratory Standards Institute (CLSI) [18]. Susceptibility of tigecycline was determined by the use of minimum inhibitory concentration (MIC) break points approved by the US Food and Drug Administration (US-FDA) [19]. For colistin, break points proposed by the European Committee on Antimicrobial Susceptibility Testing (EUCAST) were used because relevant break points were not available from CLSI [20]. In this study we did not perform further tests to identify the production of specific types of carbapenemases or other mechanisms conferring carbapenem resistance.

2.4. Data Collection. The patient's location was identified and we collected the data prospectively using a structured data sheet. Information collected included age, sex, and patient source (home or another healthcare facility). Also, extracted were duration of current hospitalization, site of infection, treatment for the index infection, and the outcome. Severity of illness was assessed by the Charlson comorbidity index (CCI) [21], hospitalization within the previous three months, patient location at the time of infection, admission to ICU, antibiotic use within three months prior to the index admission, presence of a central venous catheter (CVC), urinary catheter, mechanical ventilation, and dialysis. The presence of comorbid conditions such as cardiovascular disease, lung disease, diabetes mellitus, solid tumors or hematological malignancy, liver disease, renal failure, and chemotherapy was also documented.

The microbiological data included the causative organisms isolated from the sites of infection, the date of isolation, and the in vitro susceptibilities of the organisms to various antibiotics, including colistin and tigecycline. All collected data were independently verified by two of the authors (MAG and AAS). For patients with more than one episode of infection only data from the first episode was collected and analyzed. These cases were followed up to determine treatment received and outcome. Exposure to various risk factors was taken into consideration in the analysis only if it had occurred prior to the acquisition of the infection. Prior antibiotic exposure was considered significant for analysis only if (i) that exposure had occurred within three months prior to the index hospitalization and (ii) the antibiotic had been administered for at least 72 hours.

2.5. Statistical Analysis. Data was described as mean \pm SD and percentages. Least significant difference was measured at $95 \%$ CI. Intergroup comparison for metric variables was done by Student's $t$-test, whereas chi-square test and odds ratio were used for nonmetric variables. Binary logistic regression analysis for multivariate comparison and KaplanMeir Survival analysis predicted final outcome of the study. Analysis was performed by SPSS 22.0, JAVA stat, and MS Excel software. 
TABLE 1: Baseline demographic and clinical characteristics among the study population.

\begin{tabular}{|c|c|c|}
\hline Variables & Cases $(n=29)$ & Controls $(n=58)$ \\
\hline Mean age years \pm SD (range) & $55.4 \pm 3.8(17-85)$ & $54.7 \pm 2.6(15-94)$ \\
\hline \multicolumn{3}{|l|}{ Gender } \\
\hline Male & $18(62.1 \%)$ & $32(55.2 \%)$ \\
\hline Female & $11(37.9 \%)$ & $26(44.8 \%)$ \\
\hline \multicolumn{3}{|l|}{ Admission unit } \\
\hline Medical & $9(31.0 \%)$ & $26(44.8 \%)$ \\
\hline Surgical & $7(24.1 \%)$ & $9(15.5 \%)$ \\
\hline Intensive care & $13(44.8 \%)$ & $23(39.7 \%)$ \\
\hline \multicolumn{3}{|l|}{ Source of patient } \\
\hline Home & $22(75.9 \%)$ & $47(81.0 \%)$ \\
\hline Hospital & $7(24.1 \%)$ & $11(19.0 \%)$ \\
\hline \multicolumn{3}{|l|}{ Sites of infection } \\
\hline Bloodstream & $4(10.3 \%)$ & $7(12.1 \%)$ \\
\hline Skin and soft tissue & $7(24.1 \%)$ & $10(17.2 \%)$ \\
\hline Urinary tract & $7(27.6 \%)$ & $17(29.3 \%)$ \\
\hline Body fluid & $11(38 \%)$ & $24(41.4 \%)$ \\
\hline \multicolumn{3}{|l|}{ Infecting organisms } \\
\hline Klebsiella pneumoniae & $15(51.7 \%)$ & $31(53.4 \%)$ \\
\hline Escherichia coli & $7(24.1 \%)$ & $13(22.4 \%)$ \\
\hline Enterobacter sp. & $6(20.7 \%)$ & $12(20.7 \%)$ \\
\hline Citrobacter sp. & $1(3.5 \%)$ & $2(3.5 \%)$ \\
\hline Duration of prior hospitalization in the past 3 months (days) \pm SD (range) & $5.1 \pm 1.8(0-31)$ & $5.1 \pm 1.8(0-31)$ \\
\hline Duration of current hospitalization (days) \pm SD (range) & $56.1 \pm 16.7(0-421)$ & $26.0 \pm 5.2(0-180)$ \\
\hline Total number of comorbidities \pm SD (range) & $3.3 \pm 0.3(1-6)$ & $2.2 \pm 0.2(0-6)$ \\
\hline Number of procedures & $5.0 \pm 0.3(1-8)$ & $2.8 \pm 0.3(0-7)$ \\
\hline Carlson comorbidity index & $3.1 \pm 0.5(0-12)$ & $1.9 \pm 0.3(0-7)$ \\
\hline Mortality & $9(31.0 \%)$ & $7(12.1 \%)$ \\
\hline
\end{tabular}

2.6. Ethical Consideration. Written informed consent was obtained from all participants. The Ethics and Research Committee of KFMC approved the study.

\section{Results}

3.1. Study Population. Between March 2012 and December 2013, 29 cases of healthcare-associated infections due to CRE occurred. Fifty-eight control patients infected with CSE were consecutively selected in a ratio of $1: 2$ for cases and controls, matched for infecting organism and anatomic site of infection.

There were $62.1 \%$ and $55.2 \%$ males among the cases and controls $(p=0.540)$ with an age range of 15-94 years, mean $55.4 \pm 3.8$ (17-85 years) compared with $54.7 \pm 2.6$ (15-94 years) among the cases and controls, respectively, $p=0.065$ (Table 1). Ninety-eight percent of the studied population were of Saudi extraction. The majority of the cases and controls were admitted from home (75\% versus $81 \%$ ). Cases were admitted into medical (31\%) and surgical (24.1\%) wards, while $44.8 \%$ went to the ICU, corresponding to $44.8 \%$, $15.5 \%$, and $39.7 \%$, respectively, among the controls, $p=0.402$. However, more cases had longer hospital stay than controls $(p=0.033)$, had more comorbidities $(p=0.002)$, had undergone more procedures $(p<0.001)$, and had higher CCI $(p=0.013)$ (Table 1$)$. Among all the comorbid conditions studied, it is only renal disease requiring dialysis that was found to be independently associated with a CRE infection, $48.3 \%$ compared with (22.4\%) controls (OR 3.23 (1.24-8.39) $(p=0.014))$, Table 2 . More cases received an antibiotic in the previous three months prior to the index admission, compared with controls $(p<0.001)$.

3.2. Type of Infection. Among the cases, the isolates included Klebsiella pneumoniae (15), Escherichia coli (7), Enterobacter sp. (6), and Citrobacter sp. (1) that, respectively, caused infections in the bloodstream (4), skin and soft tissue (7), urinary tract (7), and body fluids, pleural, pericardial, peritoneal, cerebrospinal, biliary, tracheal, and abscess contents and discharges from wounds (11), while the corresponding figures among the controls were $31,13,12$, and 2 for the organisms and $7,10,17$, and 24 for the sites, respectively. There were no statistically significant differences between the cases and controls with regard to the site of infection for blood ( $p=$ $1.000)$, tissue $(p=0.444)$, urine $(p=0.611)$, and body fluids $(p=0.757)$, similarly to the infecting organism $(p=0.998)$. 
TABLE 2: Univariate analysis of risk factors associated with CRE and CSE infections.

\begin{tabular}{|c|c|c|c|c|}
\hline Characteristics & $\operatorname{CRE}(n=29)$ & $\operatorname{CSE}(n=58)$ & $\begin{array}{c}\text { Univariate } \\
\text { OR }(95 \% \mathrm{CI})\end{array}$ & $p$ value \\
\hline Female sex & $11(37.9 \%)$ & $26(44.8)$ & $0.75(0.30-1.87)$ & 0.540 \\
\hline \multicolumn{5}{|l|}{ Comorbid conditions } \\
\hline Diabetes mellitus & $18(62.1 \%)$ & $28(48.3 \%)$ & $1.75(0.71-4.36)$ & 0.224 \\
\hline Pulmonary disease & $5(17.2)$ & $13(22.4 \%)$ & $0.72(0.23-2.26)$ & 0.574 \\
\hline Renal disease & $14(48.3 \%)$ & $13(22.4 \%)$ & $3.23(1.24-8.39)$ & 0.014 \\
\hline Liver disease & $5(17.2 \%)$ & $4(6.9 \%)$ & $2.81(0.69-11.4 \%)$ & 0.153 \\
\hline Cardiovascular disease & $19(656.5 \%)$ & $27(46.6 \%)$ & $2.18(0.87-5.49)$ & 0.095 \\
\hline Malignancy & $10(34.5 \%)$ & $13(22.4 \%)$ & $1.82(0.61-4.87)$ & 0.229 \\
\hline Neurologic disease & $13(44.8 \%)$ & $20(34.5 \%)$ & $1.54(0.62-3.84)$ & 0.349 \\
\hline \multicolumn{5}{|l|}{ Clinical characteristics } \\
\hline CVC placement & $18(62.1 \%)$ & $26(44.8 \%)$ & $2.01(0.81-5.01)$ & 0.129 \\
\hline Urinary catheter & $26(89.7 \%)$ & $33(56.9 \%)$ & $6.57(1.78-24.17)$ & 0.002 \\
\hline ICU stay & $22(75.9 \%)$ & $28(48.3 \%)$ & $3.36(1.25-9.10)$ & 0.014 \\
\hline Surgery & $23(79.3 \%)$ & $31(53.4 \%)$ & $3.34(1.18-9.41)$ & 0.019 \\
\hline Mechanical ventilation & $19(65.5 \%)$ & $20(34.5 \%)$ & $3.61(1.41-9.22)$ & 0.006 \\
\hline Dialysis & $8(27.6 \%)$ & $4(6.9 \%)$ & $5.14(1.40-18.90)$ & 0.017 \\
\hline \multicolumn{5}{|l|}{ Prior antibiotic use } \\
\hline Use of any antibiotic & $18(62.1 \%)$ & $11(19 \%)$ & $6.99(2.58-18.94)$ & $<0.001$ \\
\hline Vancomycin & $4(13.8 \%)$ & $3(5.2 \%)$ & $2.93(0.61-14.10)$ & 0.215 \\
\hline Piperacillin-tazobactam & $12(41.4 \%)$ & $0(0 \%)$ & $81.9(5.73-46470974.82)$ & $<0.01$ \\
\hline Carbapenem & $9(31 \%)$ & $3(5.2 \%)$ & $8.25(2.03-33.57)$ & 0.002 \\
\hline \multicolumn{5}{|l|}{ Cephalosporin } \\
\hline First generation & $2(6.9 \%)$ & $5(8.6 \%)$ & $0.78(0.14-4.31)$ & 1.000 \\
\hline Second generation & $3(10.3 \%)$ & $1(1.7 \%)$ & $6.58(0.65-66.27)$ & 0.106 \\
\hline Third generation & $2(5.1 \%)$ & $3(5.1 \%)$ & $2.04(0.12-33.76)$ & 1.000 \\
\hline Fourth generation & $2(6.9 \%)$ & $0(0 \%)$ & $8.59(0.36-5786949.94)$ & 0.109 \\
\hline Quinolone & $9(31 \%)$ & $2(3.4 \%)$ & $12.60(2.51-63.35)$ & $<0.01$ \\
\hline Metronidazole & $8(27.6 \%)$ & $1(1.7 \%)$ & $21.71(2.56-184.23)$ & $<0.01$ \\
\hline Colistin & $1(3.4 \%)$ & $0(0 \%)$ & $4.14(0.07-3345908.39)$ & 0.333 \\
\hline
\end{tabular}

3.3. Risk Factors. By univariate analysis (Table 2) CRE infection was associated with duration of current hospitalization, antibiotic use in the previous three months, ICU stay, prior surgery, urinary catheterization, renal disease requiring dialysis, the use of any invasive procedure, and mechanical ventilation. Eighteen (62\%) of the cases had been exposed to at least one antibiotic within the previous three months, compared with 11 (19\%) of the controls $(p<0.001)$. On multivariate analysis, the duration of hospitalization, invasive procedures, and use of carbapenem were associated with infection with CRE. Not being in the ICU was protective against CRE infection (Table 3).

3.4. Outcome. Nine of 29 patients (31\%) with CRE infection died compared with 7/58 (12.1\%) of their matched controls (OR 3.28 (1.08-9.98), $p=0.031$ ), with a nonsignificant higher mortality rate among males compared with females (13 (26.0\%) versus 3 (8.1\%) OR 3.98 (0.94-19.4), $p=0.064)$. The results of the univariate and multivariate analyses of risk
TABLE 3: Multivariable analysis for the risk factors associated with CRE infection.

\begin{tabular}{lccc}
\hline Variable & OR & $95 \%$ CI & $p$ value \\
\hline $\begin{array}{l}\text { Duration of current } \\
\text { hospitalization (days) }\end{array}$ & 1.014 & $1.003-1.025$ & $\mathbf{0 . 0 1 5}$ \\
Number of procedures & 3.997 & $1.679-9.516$ & $\mathbf{0 . 0 0 2}$ \\
Carbapenem use & 20.403 & $1.769-235.379$ & $\mathbf{0 . 0 1 6}$ \\
Not being in the ICU & 0.027 & $0.001-0.496$ & $\mathbf{0 . 0 1 5}$ \\
\hline
\end{tabular}

factors for mortality are shown in Table 4. Univariate analysis revealed significant difference between the survivor and nonsurvivor subgroups, with a significantly higher percentage of the nonsurvivors being older, in the ICU on mechanical ventilation and CVP line, and received either corticosteroid, carbapenem, or tigecycline antibiotics. In addition, significantly more of the nonsurvivors had more comorbidities. However, no difference was observed in the CCI between 
TABLE 4: Univariate analysis of factors associated with 30-day mortality.

\begin{tabular}{|c|c|c|c|c|}
\hline Variable & Nonsurvivors $(n=16)$ & Survivors $(n=71)$ & OR $(95 \% \mathrm{CI})$ & $p$ value \\
\hline Age & $66.9 \pm 4.1(37,89)$ & $52.2 \pm 2.3(15,94)$ & - & 0.007 \\
\hline Gender & & & $3.98(0.94-19.40)$ & 0.064 \\
\hline Male & $13(26.0)$ & $37(74.0)$ & & \\
\hline Female & $3(8.1)$ & $34(91.9)$ & & \\
\hline Admission site & & & $9.04(2.10-44.77)$ & $<0.001$ \\
\hline ICU & $13(36.1)$ & $23(63.9)$ & & \\
\hline Medical & $1(2.9)$ & $34(97.1)$ & & \\
\hline Surgical & $2(12.5)$ & $14(87.5)$ & & \\
\hline Pulmonary disease & & & $8.86(2.28-35.86)$ & $<0.001$ \\
\hline No & $7(10.1)$ & $62(89.9)$ & & \\
\hline Yes & $9(50.0)$ & $9(50.0)$ & & \\
\hline Renal disease & & & $3.78(1.08-13.49)$ & 0.034 \\
\hline No & $7(11.7)$ & $53(88.3)$ & & \\
\hline Yes & $9(33.3)$ & $18(66.7)$ & & \\
\hline Immunotherapy & & & $5.45(1.46-20.78)$ & 0.007 \\
\hline No & $8(11.8)$ & $60(88.2)$ & & \\
\hline Yes & $8(42.1)$ & $11(57.9)$ & & \\
\hline CVP line & & & $49.14(3.64-27367830.40)$ & $<0.001$ \\
\hline No & $0(.0)$ & $43(100.0)$ & & \\
\hline Yes & $16(36.4)$ & $28(63.6)$ & & \\
\hline Foley catheter & & & $7.28(1.35-51.82)$ & 0.015 \\
\hline No & $2(7.1)$ & $26(92.9)$ & & \\
\hline Yes & $14(23.7)$ & $45(76.3)$ & & \\
\hline Stayed in ICU & & & $34.82(2.59-19367353.0)$ & $<0.001$ \\
\hline No & $0(.0)$ & $37(100.0)$ & & \\
\hline Yes & $16(32.0)$ & $34(68.0)$ & & \\
\hline Mechanical ventilation & & & $29.37(3.65-632.02)$ & $<0.001$ \\
\hline No & $1(2.1)$ & 47 (97.9) & & \\
\hline Yes & $15(38.5)$ & $24(61.5)$ & & \\
\hline Carbapenem & & & $3.79(1.08-13.49)$ & 0.034 \\
\hline No & $7(11.7)$ & $53(88.3)$ & & \\
\hline Yes & $9(33.3)$ & $18(66.7)$ & & \\
\hline Tigecycline & & & $10.30(1.77-65.67)$ & 0.004 \\
\hline No & $11(13.9)$ & $68(86.1)$ & & \\
\hline Yes & $5(62.5)$ & $3(37.5)$ & & \\
\hline
\end{tabular}

those that survived and those who did not $(p=0.857)$. The control subjects had more chances of survival as shown by the Kaplan-Meier curve $p=0.013$ (Figure 1). Cumulative survival time was shorter among the cases than controls ((22.4\% versus $80.6 \%)$ OR 3.28 (0.95-11.55)). None of these variables, however, showed any significance when subjected to multivariable analysis.

The majority of the cases were treated with a colistinbased regimen (55\%), either alone (68.8\%) or in combination with tigecycline $(31.2 \%)$. Among those who received any colistin-based regimen, $50 \%$ died, segregated by colistin only (45.5\%) and colistin-tigecycline combination (60\%). Other antibiotics used in the treatment of the CRE included ciprofloxacin (24\%), tigecycline (10.5\%), amikacin (6.9\%), and nitrofurantoin $(3.6 \%)$.

\section{Discussion}

Carbapenem resistance among the Enterobacteriaceae is an emerging phenomenon of vast clinical and public health importance. This study was necessitated by an observation of a rising trend of infections due to multidrug resistant Gramnegative (MDR GN) pathogens in our institution [15]. Recent studies from other Middle Eastern countries also revealed reduced susceptibility of K. pneumoniae to carbapenems [22, 23]. However, this is the first case-control study addressing this topic among Enterobacteriaceae from the region.

Antibiotic resistance among hospitalized patients remains a major global public health problem with attendant increase in healthcare costs, as well as treatment failures, in addition to mortality $[2,10-12]$. Carbapenems are often the 


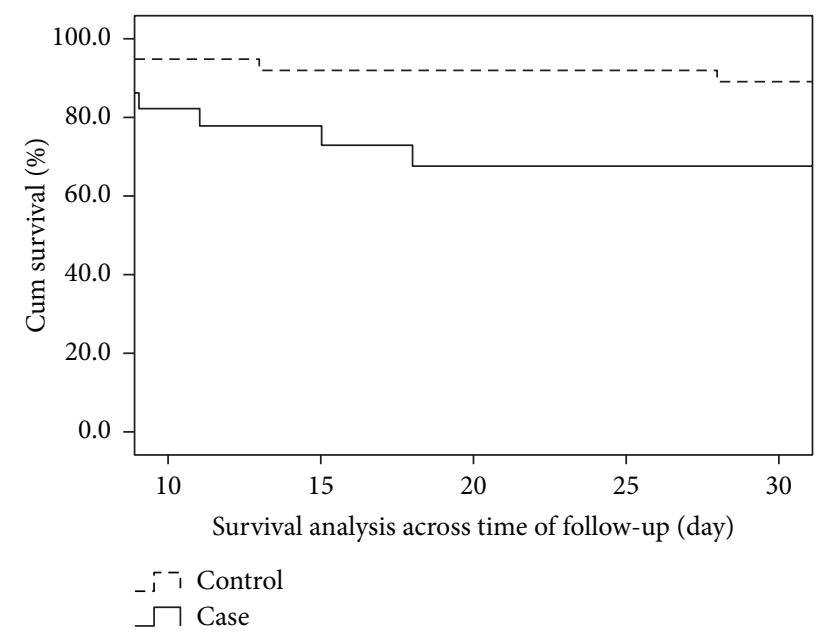

FIGURE 1: Survival curve using the Kaplan-Meier method for cases compared with controls. The cases $(-)$ had a lower probability of survival than the controls $(--)(p=0.013)$.

antibiotics of last resort to treat infections due to extended spectrum $\beta$-lactamase- (ESBL) or plasmid-mediated AmpC(pAmpC-) producing organisms. These pathogens are frequently also resistant to other antibiotic classes including quinolones, aminoglycosides, and trimethoprim-sulfamethoxazole [10-12]. The increasing prevalence of CRE, especially during the past 10 years, has seriously compromised the therapeutic armamentarium against infections due to these organisms [2].

Previous studies reported similar risk factors for carbapenem resistant $K$. pneumoniae infection and demonstrated associations with length of hospital stay, ICU admission, use of CVC, recent solid-organ or stem-cell transplantation, receipt of mechanical ventilation, and exposure to broad-spectrum antibiotics $[3,7,8]$. Variables found to be associated with CRE infection in our study include the use of urinary catheter, mechanical ventilation, dialysis in addition to ICU stay, and surgery. Among antibiotics, prior exposure to piperacillin-tazobactam, carbapenem, quinolone, and metronidazole was associated with infection with CRE. Contrary to our finding, Falagas et al. [8] reported that exposure to anti-pseudomonal antibiotics was not associated with CRE infection. Not being in the ICU is protective against CRE infection. These findings are in agreement with previous studies [24-26]. Ho et al. [27] however reported no significant increase in the rate of carbapenem resistance among their patients during the period 2006 to 2010 despite a significant increase in consumption. Recent studies from Saudi Arabia [28] and other Gulf countries [29] showed that OXA-48 and NDM-1 are the dominant carbapenemases among Enterobacteriaceae with low prevalence of VIM. Klebsiella pneumoniae accounts for the largest proportion of infections among our cases and controls (51.7\% versus 53.4\%, resp.), with the least common isolate being Citrobacter spp. (3.5\% in both groups). This was corroborated by a recent study from Asian countries between 2000 and 2012 [30] where K. pneumoniae was reported to be the leading cause of nosocomial infections (39.3\%), while Citrobacter spp. caused the least (4.5\%).
The optimal treatment of infection due to CRE is uncertain, and antibiotic options are limited. The presence of a KPC or metalloenzyme carbapenemase confers resistance to all commonly used antibiotics [31]; hence antimicrobial susceptibility results for agents outside the $\beta$-lactam and carbapenem classes guide the selection of antibiotic therapy in such cases. This calls for the expansion of the antibiograms used in most centers, which ordinarily do not include agents like colistin or polymyxin B, tigecycline, aztreonam, and fosfomycin (especially for urinary isolates) [32]. The use of two or more antimicrobial agents in combination for the treatment of CRE has been in practice despite limited clinical data because of the high mortality associated with monotherapy. Clinical evidence suggests that treatment with combination therapy may improve outcome [33-38]. Significantly more treatment failures were reported among cases treated with monotherapy compared with combination as published in a recent review of 38 articles that included case reports and case series ( $49 \%$ versus $25 \%, p=0.01$ ) [39]. In a retrospective review of 125 patients with bacteremia due to KPC gene-harboring K. pneumoniae the overall mortality rate at 30 days was $42 \%$ [35]. The mortality rate was lower among patients who received combination therapy with two or more drugs (27/79 (34\%)) compared with monotherapy with colistin, tigecycline, or gentamicin (25/46 (54\%)). Patients treated with a combination of a polymyxin plus tigecycline had a mortality rate of 30 percent ( 7 of 23 ), while the regimen of colistin, tigecycline, and extended-infusion meropenem (a dose of 2 grams infused over three or more hours every eight hours) was associated with the lowest mortality rate (2 of $16(12.5 \%)$ ). Colistin, tigecycline, aminoglycosides, and carbapenems were considered in the prescribed regimen even if the antibiogram showed resistant isolates.

A systematic review of 20 observational studies also concluded that combination therapy may offer a survival advantage in severely ill patients [38]. Colistin formed the backbone of the treatment received by our patients, with $55 \%$ receiving a colistin-based regimen. Out of these, $68.8 \%$ were treated with colistin only, while $31.2 \%$ received a combination of colistin and tigecycline, whereas ciprofloxacin (24.2\%), tigecycline $(10.3 \%)$, amikacin $(6.9 \%)$, and nitrofurantoin $(3.6 \%)$ were some of the monotherapy regimens in our cohort. These encouraging results from the use of combination therapy have made physicians, mainly out of desperation, adopt the practice as standard of care in the treatment of CRE infections.

We found a significantly higher mortality rate among those with CRE infection $(p=0.031)$. From a recent review by Falagas et al. [38] the use of combination antimicrobials like colistin with tigecycline, colistin with carbapenem, and tigecycline with gentamicin might result in lower mortality than with other combinations of antibiotics, although this ranged between $50 \%$ and $60 \%$ for patients in critical care and non-ICU settings, respectively. This figure is similar to ours where mortality was $50 \%$ among those who received colistin either alone or in combination. Recently, Vardakas et al. [39] showed that the triple combination of tigecycline, colistin, and an aminoglycoside in the treatment of CRKP had lower mortality $(2 / 8,25 \%)$ compared with any other combination 
$(p<0.002)$. With the increased use of colistin and tigecycline to treat infections due to $\mathrm{CRE}$, resistance to these lifesaving medications has emerged [40-43].

\section{Limitations}

Our study has limitations. In addition to the relatively small number of patients, matching control patients to cases was a challenge due to the rarity of some of the organisms isolated. Being a single-center study, these results may not be generalizable to other centers where different factors might be contributing to similar infections. However, we thought that we should share our experience with these emerging and increasingly resistant organisms and assess the likely factors that could be contributory to the acquisition of infections due to $\mathrm{CRE}$ and describe their treatment and outcome.

\section{Conclusions}

From this single-center observational case-control study we have identified duration of index admission, prior antibiotic use, ICU stay, and invasive procedures to be independently associated with CRE infection. Infection with CRE was also associated with higher mortality compared with CSE. Available evidence from nonrandomized studies, as well as from our limited experience at our center, suggests that combination antibiotic treatment may offer a comparative survival advantage over monotherapy. Improved hand hygiene, barrier nursing, continuous education, minimizing device use, enhanced surveillance, and antimicrobial stewardship will limit CRE transmission in healthcare facilities. Welldesigned randomized controlled trials are required to address this crucial question of everyday clinical practice as we await the development of novel antimicrobial agents with reliable efficacy against MDR GNR.

\section{Competing Interests}

The authors declare that they have no competing interests.

\section{Acknowledgments}

The authors appreciate the contributions of the following staff of King Fahad Medical City to this project: Professor Imad Tleyjeh, Infectious Diseases Section, Medical Specialties Department; Professor Omar Kasule, Faculty of Medicine; Ms. Abeer, microbiology laboratory; and Mr. Tareq Wani, Research and Scientific Publication Center. This research was made possible with the help of an intramural research fund from King Fahad Medical City (IRF 012-026).

\section{References}

[1] K. M. Papp-Wallace, A. Endimiani, M. A. Taracila, and R. A. Bonomo, "Carbapenems: past, present, and future," Antimicrobial Agents and Chemotherapy, vol. 55, no. 11, pp. 4943-4960, 2011.
[2] K. Z. Vardakas, D. K. Matthaiou, M. E. Falagas, E. Antypa, A. Koteli, and E. Antoniadou, "Characteristics, risk factors and outcomes of carbapenem-resistant Klebsiella pneumoniae infections in the intensive care unit," Journal of Infection, vol. 70, no. 6, pp. 592-599, 2015.

[3] M. Souli, I. Galani, A. Antoniadou et al., "An outbreak of infection due to $\beta$-lactamase Klebsiella pneumoniae carbapenemase 2-producing $K$. pneumoniae in a Greek university hospital: Molecular characterization, epidemiology, and outcomes," Clinical Infectious Diseases, vol. 50, no. 3, pp. 364-373, 2010.

[4] F. Baquero, M. C. Negri, M. I. Morosini, and J. Blázquez, "Antibiotic-selective environments," Clinical Infectious Diseases, vol. 27, supplement 1, pp. S5-S11, 1998.

[5] Centers for Disease Control and Prevention (CDC), "Vital signs: carbapenem-resistant Enterobacteriaceae," Morbidity and Mortality Weekly Report, vol. 62, no. 9, pp. 165-170, 2013.

[6] W. C. Rottier, H. S. M. Ammerlaan, and M. J. M. Bonten, "Effects of confounders and intermediates on the association of bacteraemia caused by extended-spectrum $\beta$-lactamase-producing Enterobacteriaceae and patient outcome: a meta-analysis," Journal of Antimicrobial Chemotherapy, vol. 67, no. 6, pp. 1311-1320, 2012.

[7] G. Patel, S. Huprikar, S. H. Factor, S. G. Jenkins, and P. Calfee, "Outcomes of carbapenem resistant Klebsiella pneumoniae infection and impact of antimicrobial and adjunctive therapies," Infection Control \& Hospital Epidemiology, vol. 29, pp. 10991106, 2008.

[8] M. E. Falagas, P. I. Rafailidis, D. Kofteridis et al., "Risk factors of carbapenem-resistant Klebsiella pneumoniae infections: a matched case-Control study," Journal of Antimicrobial Chemotherapy, vol. 60, no. 5, pp. 1124-1130, 2007.

[9] A. Borer, L. Saidel-Odes, K. Riesenberg et al., "Attributable mortality rate for carbapenem-resistant Klebsiella pneumoniae bacteremia," Infection Control and Hospital Epidemiology, vol. 30, no. 10, pp. 972-976, 2009.

[10] C. J. Gregory, E. Llata, N. Stine et al., "Outbreak of carbapenemresistant Klebsiella pneumoniae in Puerto Rico associated with a novel carbapenemase variant," Infection Control and Hospital Epidemiology, vol. 31, no. 5, pp. 476-484, 2010.

[11] K. Hussein, H. Sprecher, T. Mashiach, I. Oren, I. Kassis, and R. Finkelstein, "Carbapenem resistance among Klebsiella pneumoniae isolates: risk factors, molecular characteristics, and susceptibility patterns," Infection Control and Hospital Epidemiology, vol. 30, no. 7, pp. 666-671, 2009.

[12] K. Hussein, A. Raz-Pasteur, R. Finkelstein et al., "Impact of carbapenem resistance on the outcome of patients' hospitalacquired bacteraemia caused by Klebsiella pneumoniae," Journal of Hospital Infection, vol. 83, no. 4, pp. 307-313, 2013.

[13] P. Nordmann, T. Naas, and L. Poirel, "Global spread of carbapenemase producing Enterobacteriaceae," Emerging Infectious Diseases, vol. 17, no. 10, pp. 1791-1798, 2011.

[14] R. Cantón, M. Akóva, Y. Carmeli et al., "Rapid evolution and spread of carbapenemases among Enterobacteriaceae in Europe," Clinical Microbiology and Infection, vol. 18, no. 5, pp. 413-431, 2012.

[15] M. A. Garbati, A. Bin Abdulhak, K. Baba, and H. Sakkijha, "Infection due to colistin-resistant Enterobacteriacae in critically-ill patients," Journal of Infection in Developing Countries, vol. 7, no. 10, pp. 713-719, 2013. 
[16] T. Tängdén and C. G. Giske, "Global dissemination of extensively drug-resistant carbapenemase-producing Enterobacteriaceae: clinical perspectives on detection, treatment and infection control," Journal of Internal Medicine, vol. 277, no. 5, pp. 501-512, 2015.

[17] S. Vergara-López, M. C. Domínguez, M. C. Conejo, Á. Pascual, and J. Rodríguez-Baño, "Lessons from an outbreak of metallo$\beta$-lactamase-producing Klebsiella oxytoca in an intensive care unit: the importance of time at risk and combination therapy," The Journal of Hospital Infection, vol. 89, no. 2, pp. 123-131, 2015.

[18] Clinical and Laboratory Standard Institute, "Performance standard for antimicrobial susceptibility testing: twenty-second informational supplement," Tech. Rep. M100-S23, CLSI, Wayne, Pa, USA, 2013.

[19] United States Food and Drug Administration (FDA), "Highlights of prescribing information Tygacil," 2014, http://www.fda .gov/safety/medwatch/safetyinformation/safety-relateddruglabelingchanges/ucm132714.

[20] EUCAST, January 2012, http://www.eucast.org/fileadmin/src/ media/PDFs/EUCAST_files/Disk_test_documents/EUCAST _breakpoints_v_2.0_111130.pdf.

[21] M. E. Charlson, P. Pompei, K. L. Ales, and C. R. MacKenzie, "A new method of classifying prognostic comorbidity in longitudinal studies: development and validation," Journal of Chronic Diseases, vol. 40, no. 5, pp. 373-383, 1987.

[22] H. H. Balkhy, A. El-Saed, S. M. Al Johani et al., "The epidemiology of the first described carbapenem-resistant Klebsiella pneumoniae outbreak in a tertiary care hospital in Saudi Arabia: How far do we go?" European Journal of Clinical Microbiology \& Infectious Diseases, vol. 31, no. 8, pp. 1901-1909, 2012.

[23] A. Shibl, M. Al-Agamy, Z. Memish, A. Senok, S. A. Khader, and A. Assiri, "The emergence of OXA-48- and NDM-1-positive Klebsiella pneumoniae in Riyadh, Saudi Arabia," International Journal of Infectious Diseases, vol. 17, no. 12, pp. el130-e1133, 2013.

[24] M. Swaminathan, S. Sharma, S. P. Blash et al., "Prevalence and risk factors for acquisition of carbapenem-resistant Enterobacteriaceae in the setting of endemicity," Infection Control and Hospital Epidemiology, vol. 34, no. 8, pp. 809-817, 2013.

[25] M.-H. Jeon, S.-H. Choi, Y. G. Kwak et al., "Risk factors for the acquisition of carbapenem-resistant Escherichia coli among hospitalized patients," Diagnostic Microbiology and Infectious Disease, vol. 62, no. 4, pp. 402-406, 2008.

[26] A. S. Chitnis, P. S. Caruthers, A. K. Rao et al., "Outbreak of carbapenem-resistant Enterobacteriaceae at a long-term acute care hospital: sustained reductions in transmission through active surveillance and targeted interventions," Infection Control and Hospital Epidemiology, vol. 33, no. 10, pp. 984-992, 2012.

[27] C.-M. Ho, M.-W. Ho, Y.-C. Liu et al., "Correlation between carbapenem consumption and resistance to carbapenems among Enterobacteriaceae isolates collected from patients with intraabdominal infections at five medical centers in Taiwan, 20062010," International Journal of Antimicrobial Agents, vol. 40, supplement 1, pp. S24-S28, 2012.

[28] Z. A. Memish, A. Assiri, M. Almasri et al., "Molecular characterization of carbapenemase production among gram-negative bacteria in Saudi Arabia," Microbial Drug Resistance, vol. 21, no. 3, pp. 307-314, 2015.

[29] H. M. Zowawi, A. L. Sartor, H. H. Balkhy et al., "Molecular characterization of carbapenemase-producing Escherichia coli and Klebsiella pneumoniae in the countries of the gulf cooperation council: dominance of OXA-48 and NDM Producers," Antimicrobial Agents and Chemotherapy, vol. 58, no. 6, pp. 3085-3090, 2014.

[30] Y. Xu, B. Gu, M. Huang et al., "Epidemiology of carbapenem resistant Enterobacteriaceae (CRE) during 2000-2012 in Asia," Journal of Thoracic Disease, vol. 7, no. 3, pp. 376-385, 2015.

[31] M. Castanheira, H. S. Sader, L. M. Deshpande, T. R. Fritsche, and R. N. Jones, "Antimicrobial activities of tigecycline and other broad-spectrum antimicrobials tested against serine carbapenemase- and metallo- $\beta$-lactamase-producing Enterobacteriaceae: report from the SENTRY antimicrobial surveillance program," Antimicrobial Agents and Chemotherapy, vol. 52, no. 2, pp. 570-573, 2008.

[32] D. M. Livermore, M. Warner, S. Mushtaq, M. Doumith, J. Zhang, and N. Woodford, "What remains against carbapenemresistant Enterobacteriaceae? Evaluation of chloramphenicol, ciprofloxacin, colistin, fosfomycin, minocycline, nitrofurantoin, temocillin and tigecycline," International Journal of Antimicrobial Agents, vol. 37, no. 5, pp. 415-419, 2011.

[33] Z. A. Qureshi, D. L. Paterson, B. A. Potoski et al., "Treatment outcome of bacteremia due to KPC-producing Klebsiella pneumoniae: superiority of combination antimicrobial regimens," Antimicrobial Agents and Chemotherapy, vol. 56, no. 4, pp. 21082113, 2012.

[34] O. Zarkotou, S. Pournaras, P. Tselioti et al., "Predictors of mortality in patients with bloodstream infections caused by KPCproducing Klebsiella pneumoniae and impact of appropriate antimicrobial treatment," Clinical Microbiology and Infection, vol. 17, no. 12, pp. 1798-1803, 2011.

[35] E. B. Hirsch and V. H. Tam, "Detection and treatment options for Klebsiella pneumoniae carbapenemases (KPCs): an emerging cause of multidrug-resistant infection," Journal of Antimicrobial Chemotherapy, vol. 65, no. 6, Article ID dkq108, pp. 1119$1125,2010$.

[36] M. Tumbarello, P. Viale, C. Viscoli et al., "Predictors of mortality in bloodstream infections caused by Klebsiella pneumoniae carbapenemase-producing K. pneumoniae: importance of combination therapy," Clinical Infectious Diseases, vol. 55, no. 7, pp. 943-950, 2012.

[37] F. Sbrana, P. Malacarne, B. Viaggi et al., "Carbapenem-sparing antibiotic regimens for infections caused by Klebsiella pneumoniae carbapenemase-producing K. pneumoniae in intensive care unit," Clinical Infectious Diseases, vol. 56, no. 5, pp. 697-700, 2013.

[38] M. E. Falagas, P. Lourida, P. Poulikakos, P. I. Rafailidis, and G. S. Tansarli, "Antibiotic treatment of infections due to carbapenemresistant Enterobacteriaceae: systematic evaluation of the available evidence," Antimicrobial Agents and Chemotherapy, vol. 58, no. 2, pp. 654-663, 2014.

[39] K. Z. Vardakas, D. K. Matthaiou, M. E. Falagas, E. Antypa, A. Koteli, and E. Antoniadou, "Tigecycline for carbapenemresistant Klebsiella pneumoniae infections in the intensive care unit," Infectious Diseases, vol. 47, no. 10, pp. 751-753, 2015.

[40] S. Pournaras, G. Vrioni, E. Neou et al., "Activity of tigecycline alone and in combination with colistin and meropenem against Klebsiella pneumoniae carbapenemase (KPC)-producing Enterobacteriaceae strains by time-kill assay," International Journal of Antimicrobial Agents, vol. 37, no. 3, pp. 244-247, 2011.

[41] A. Antoniadou, F. Kontopidou, G. Poulakou et al., "Colistinresistant isolates of Klebsiella pneumoniae emerging in intensive 
care unit patients: first report of a multiclonal cluster," Journal of Antimicrobial Chemotherapy, vol. 59, no. 4, pp. 786-790, 2007.

[42] D. Marchaim, T. Chopra, J. M. Pogue et al., "Outbreak of colistin-resistant, carbapenem-resistant Klebsiella pneumoniae in Metropolitan Detroit, Michigan," Antimicrobial Agents and Chemotherapy, vol. 55, no. 2, pp. 593-599, 2011.

[43] J. Lee, G. Patel, S. Huprikar, D. P. Calfee, and S. G. Jenkins, "Decreased susceptibility to polymyxin B during treatment for carbapenem-resistant Klebsiella pneumoniae infection," Journal of Clinical Microbiology, vol. 47, no. 5, pp. 1611-1612, 2009. 


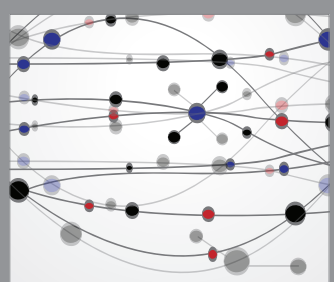

The Scientific World Journal
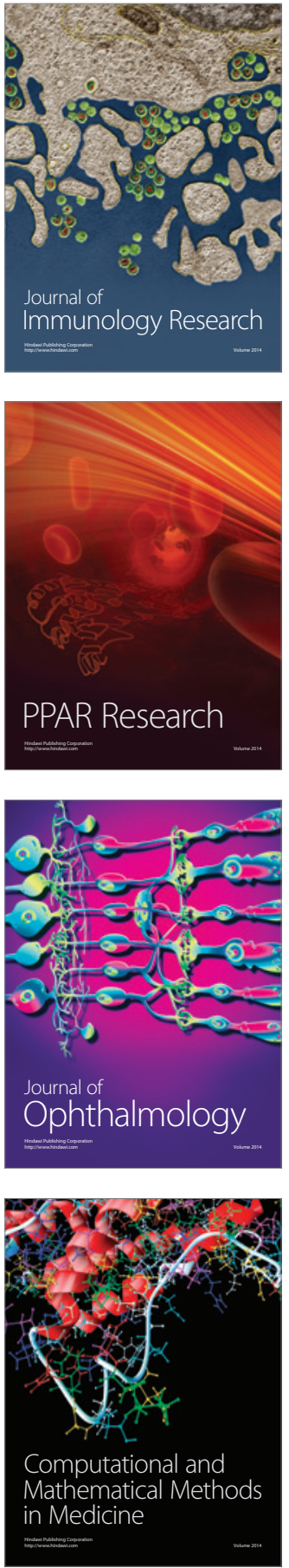

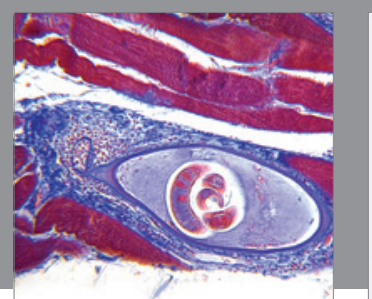

Gastroenterology Research and Practice

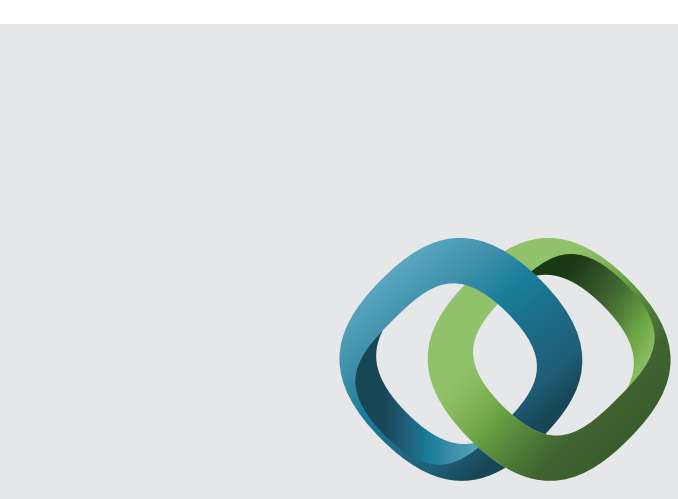

\section{Hindawi}

Submit your manuscripts at

http://www.hindawi.com
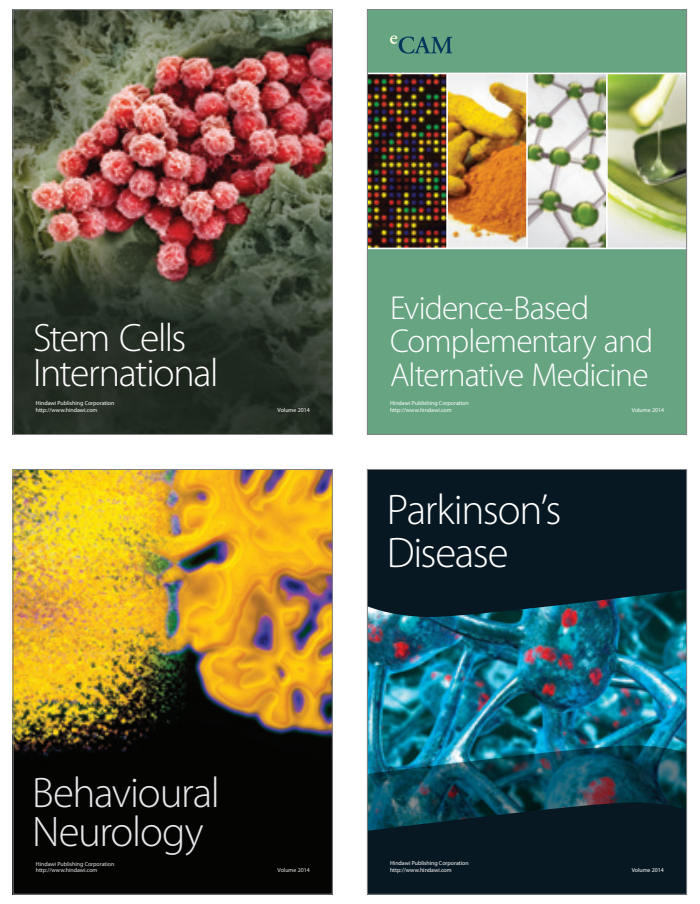
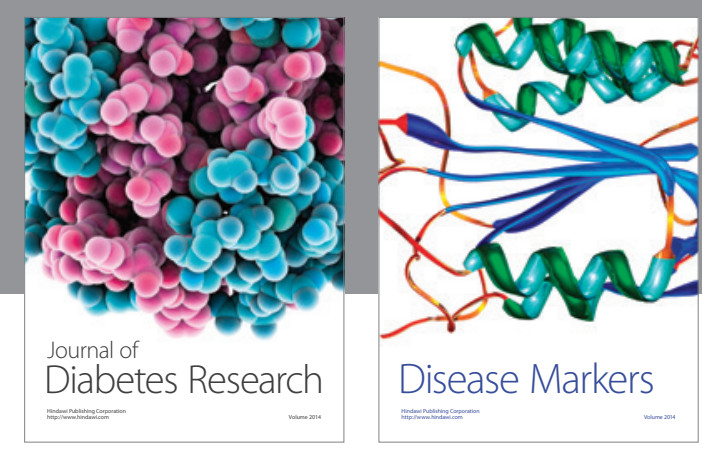

Disease Markers
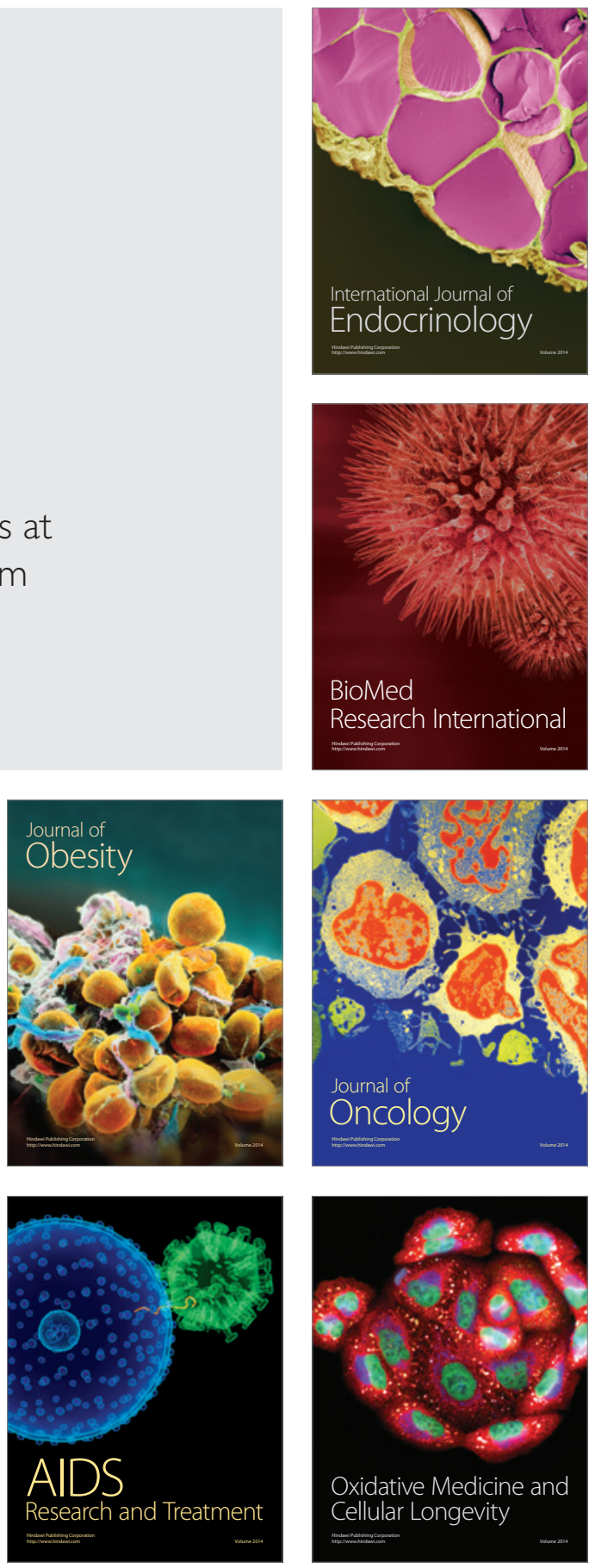